Revue d'histoire de l'Amérique française

REVUE D.HISTOIRE DE L'AMÉRIQUE FRANÇAISE

\title{
L'emplacement du fort de Dollard des Ormeaux
}

\section{Victor Morin}

Volume 6, numéro 1, juin 1952

URI : https://id.erudit.org/iderudit/301500ar

DOI : https://doi.org/10.7202/301500ar

Aller au sommaire du numéro

Éditeur(s)

Institut d'histoire de l'Amérique française

ISSN

0035-2357 (imprimé)

1492-1383 (numérique)

Découvrir la revue

Citer cet article

Morin, V. (1952). L'emplacement du fort de Dollard des Ormeaux. Revue

d'histoire de l'Amérique française, 6(1), 3-19. https://doi.org/10.7202/301500ar d'utilisation que vous pouvez consulter en ligne.

https://apropos.erudit.org/fr/usagers/politique-dutilisation/ 


\section{L'EMPLACEMENT DU FORT DE DOLLARD DES ORMEAUX*}

Les récits historiques et les traditions de trois siècles d'existence ont établi de façon précise à Carillon, sur la rive nord de la Rivière Ottawa, le théâtre du merveilleux exploit de Dollard des Ormeaux et de ses compagnons, de même qu'ils ont clairement démontré que c'est grâce à leur généreux sacrifice que l'établissement encore chancelant de la France en Amérique a pu survivre.

Et pourtant! Combien de fois n'a-t-on pas tenté de discréditer le geste de ces sauveurs de la Nouvelle-France? Un professeur d'université anglaise, en veine d'humour funambulesque, n'est-il pas allé jusqu'à dire que "Dollard devrait occuper une place importante au musée des mythes historiques?" Cette tentative de démolition n'a pas manqué d'être aussitôt relevée dans les milieux mêmes où elle avait été lancéé ${ }^{1}$.

Un autre, professeur de collège classique celui-là, aurait avancé de son côté, qu'il ne fallait pas trop se fier "au témoignage d'un seul survivant, un pauvre Huron sorti de l'aventure plus qu'à moitié ébranlé d'esprit'. Aurait-il voulu prétendre, par hasard, que Dollard et ses compagnons seraient sortis indemnes de leur tragique aventure et qu'au lieu de revenir dans leurs foyers, iis seraient allés s'établir tranquillement à la campagne sous des noms d'emprunt?

* Beaucoup de nos lesteurs nous avaient demandé notre sentiment sur les récentes découvertes de géologues sur la ferme Ross, cinq milles à l'est de Hawkesbury (Ontario). D'aucuns ont même profité de ces découvertes pour remettre en question le fait Dollard. L'un de nos membres honoraires nous a apporté les pages que l'on va lire. M. Victor Morin, chercheur émérite toute sa vie, connaít bien l'histoire du Montréal des premiers temps. Nous estimons son étude concluante. (Le Directeur de la Revue).

1. Voir les articles du Montreal Herald en date du 21 mars et du 8 avril et celui de la Montreal Gazette du 2 avril 1932, et les répliques d'Emile Vaillancourt et de W.H. Atherton dans les mêmes journaux le 26 mars pour le Herald et les 29 mars et 7 avril pour la Gazette. 
D'autres enfin, devant l'impossibilité de nier la sanglante épopée, ont tenté d'en salir la générosité en prétendant que le but des dix-sept combattants qui avaient ainsi résolu de courir à la mort en face de trois cents Iroquois, se réduisait à un complot de pillage de fourrures au détriment d'honnêtes trafiquants! Pourquoi n'ontils pas en même temps accusé le fondateur et gouverneur de VilleMarie, qui avait autorisé la tentative de cet exploit désespéré, d'une sordide complicité de profiteur dans un tel coup de main?

L'abbé Lionel Groulx a fait bonne justice de ces élucubrations dans divers articles de journaux ${ }^{2}$, et dans le Dossier de Dollard publié aussitôt après; mais, comme l'hydre de Lerne, la calomnie a des têtes sans cesse renaissantes qu'il faut s'empresser d'abattre si l'on veut éviter d'en être victime.

La dernière tentative en cette matière a pour but de déplacer au moins le théâtre de l'exploit de Dollard au profit de la province sœur d'Ontario; elle s'appuie sur les travaux d'hommes de science à qui le Service Géologique du Canada a confié la mission de faire des fouilles sur la ferme Ross, située au sud de la Rivière Ottawa, à cinq milles en aval de Hawkesbury dans la province d'Ontario, à sept milles en dehors de ia frontière du Québec, en face de l'endroit connu sous le nom de Greece Point de l'autre côté de la rivière, parce qu'une vieille sauvagesse aurait raconté "qu'un terrible combat avait eu lieu à cet endroit entre les Français et les gens de sa tribu il y $a$ très longtemps". Les recherches de ces savants sont très intéressantes au point de vue technique; poursuivies jusqu'au mois de novembre 1951, elles ont été minutieusement consignées dans un rapport dont nous avons eu l'avantage de prendre connaissance avant sa publication prochaine, mais qui a le tort capital de conclure hâtivement qu'il s'agit ici du Fort de Dollard des Ormeaux, sans appuyer cette hypothèse sur quelque document que ce soit. On procède par simple supposition gratuite, alors que les relations et autres documents historiques contredisent absolument cet avancé. Les vagues racontars d'une Indienne ignare auraient-ils préséance sur les écrits d'historiens sérieux et sur les traditions religieusement transmises de siècle en siècle dans les milieux véritablement intéressés, surtout quand on songe que les combats entre Indiens et

2. Volr en particulier le Devoir en date des 7 et 11 mai 1932. 
Faces Pâles, et la construction de palissades défensives, ont eu lieu un peu partout dans l'étendue de notre pays?

Garneau, Ferland, Faillon, Parkman, Sulte, Fauteux, Massicotte, Atherton et autres écrivains de mérite ont fait depuis longtemps justice de telles billevesées en s'appuyant sur les témoignages de documents indiscutables. L'abbé Guindon a confirmé leurs conclusions sur des relevés hydrographiques du Long-Sault, en fixant de façon irréfutable le theâtre du merveilleux exploit à Carillon, sur la rive nord de la Rivière Ottawa, seul endroit où se trouvait le "passage infaillible" que les coureurs de bois et les Indiens devaient nécessairement suivre en descendant des "pays d'en haut"; dès 1865, l'abbé Faillon exprimait l'espoir qu'un monument s'élèverait bientôt à Montréal à la gloire de Dollard et de ses compagnons et Benjamin Sulte, l'un des plus érudits de nos historiens, écrivait en 1891, dans ses pages d'Histoire du Canada, au sujet du siège du LongSault; "nous avons parcouru ces lieux et tout nous dit que le coteau de Carillon est le piédestal d'une statue qui sera dressée un jour et qui manque à cette belle partie de l'Ottawa ${ }^{3}$."

On savait à Québec et à Montréal que, depuis 1658, les Iroquois préparaient une invasion massive de la Nouvelle-France afin de détruire pour toujours cet établissement français. Dès cette année, la colonie française d'Onnontagué, établie deux ans auparavant dans le but d'assurer une paix durable avec les Cinq Cantons, s'évadait pendant la nuit afin d'échapper à un massacre imminent et $\mathbf{M}$. de Maisonneuve édictait une ordonnance défendant aux habitants de s'éloigner en excursion de chasse ou de pêche afin d'éviter les surprises d'un ennemi constamment en embuscade. Au cours des deux années suivantes, les Iroquois dont on s'empare et qu'on condamne au bûcher, défient leurs bourreaux et déclarent avec jactance qu'une campagne est en voie de préparation pour attaquer la colonie sur divers points et détruire de fond en comble les établissements de Québec, Trois-Rivières et Montréal ${ }^{4}$.

3. Ce monument s'élève aujourd'hui sur le parc Lafontaine à Montréal et un buste de Dollard a été érigé sur piédestal à Carillon près de la rivière Ottawa, sur un terrain fourni par la Fabrique de cette paroisse qui s'est chargé? de son entretien par acte du 24 mai 1919 .

4. Voir Lettres de la Mère Marie de l'Incarnation à son fils Dom Claude Martin en date du 25 juin 1660 . 
C'est dans le but de faire échec à ces desseins que Dollard et ses compagnons offrent le sacrifice de leurs vies. Après mûre réflexion, M. de Maisonneuve admire la hardiesse de ce projet et lui donne son approbation, chose qu'il aurait absolument refusée, dans les circonstances, s'il s'était agi d'une embuscade ordinaire et encore plus si l'expédition avait eu pour but de s'emparer d'un convoi de fourrures, ainsi que certains écrivains malveillants ont voulu le faire entendre.

On connaît la suite des événements et les résultats qui les ont suivis. Le premier récit de l'hécatombe du Long-Sault est apporté à Montréal le 3 juin 1660 par le Huron Louis, un des acteurs et transfuges du drame, qui avait réussi à s'échapper des mains des vainqueurs. Et c'est sur la foi de ces renseignements que le Curé Souart inscrit le nécrologe de ses dix-sept paroissiens au registre de Montréal. Deux autres Hurons également échappés de captivité viennent plus tard confirmer ce récit et le Père jésuite Chaumonot en recueille les détails dans une lettre dont la Rév. Mère Marie de l'Incarnation s'inspire à son tour pour en envoyer la relation à son fils en France; elle y revient dans une autre lettre du 2 novembre suivant en ajoutant la confirmation des faits apportés par un Huron chrétien dont le témoignage appuie les précédents.

De son côté, le Père Lallemant, supérieur de la Mission des Jésuites à Québec, relate au long l'épopée de Dollard et de ses compagnons dans la Relation de 1659-1660 envoyée au Supérieur de sa Communauté en France suivant la coutume établie depuis 1632, en réunissant les témoignages de trois autres Hurons également acteurs du drame et transfuges à l'ennemi, que la modestie empêche cependant de fournir ce dernier détail. Marguerite Bourgeoys, fondatrice de la Congrégation de Notre-Dame de Montréal, apporte son témoignage au geste patriotique de Dollard qui "assembla seize ou dix sept hommes des plus généreux pour aller attaquer les Sauvages à dessein d'y donner leur vie si c'était la volonté de Dieu.".

Quelques jours s'étaient à peine écoulés depuis le combat, que Pierre-Esprit Radisson, ce coureur de bois deux fois transfuge de France en Angleterre, passait sur le thêâtre de la tragique héca-

5. C. Faillon, Histoire de la Colonie Frangaise en Canada (3 vol., Villemarie, 1865), II: 414. 
tombe en revenant du lac Michigan et qu'il en relevait les traces encore fraiches dans les allées et venues des bandes iroquoises le long de la Rivière Ottawa ${ }^{6}$.

Mais la version la plus authentique de ce fait d'armes, digne de figurer dans les fastes de l'humanité, nous est fournie par le sulpicien Dollier de Casson, auteur d'une Histoire du Montréal, écrite en 1673 et publiée par la Société Historique de cette ville en 1869. Arrivé à Ville-Marie en 1666, soit six ans seulement après l'exploit du Long-Sault, cet historien a pu en interroger les nombreux contemporains encore vivants à cette époque et notamment le curé Souart, Jeanne Mance, Marguerite Bourgeoys, le notaire Benigne Basset qui avait dressé le testament de l'un des combattants, les notables Charles LeMoyne, Zacharie Dupuis, et surtout quelques-uns des Hurons qui avaient participé au drame. En poursuivant son enquête de côté et d'autre, il a pu contrôler l'exactitude des renseignements recueillis jusqu'alors et le caractère de cet ancien officier des armées de Turenne nous permet d'accorder toute confiance à son récit.

L'abbé Vachon de Belmont vint à son tour habiter Montréal en 1680. Les beautés de l'histoire de cette bourgade chrétienne et civilisatrice perdue en avant-garde au centre de la barbarie indienne et la grandeur des faits d'armes accomplis par ses pionniers le séduirent à tel point qu'il entreprit d'en écrire à son tour une histoire à l'aide des documents recueillis depuis un demi siècle? ${ }^{7}$. Il eut la bonne fortune de prendre contact avec les chefs des diverses tribus indiennes réunies à Ville-Marie par le Gouverneur de Callị̀res en 1700 et 1701 pour la signature d'un traité de paix générale et c'est lui qui apprit de l'Iroquois Taondechoren, grâce à sa connaissance de langues indiennes, que le combat du Long-Sault avait coûté aux assiégeants le tiers de leurs guerriers.

A son tour, le sulpicien Faillon a réuni ces diverses sources historiques auxquelles il a ajouté ses recherches personnelles dans les

6. Le Mémoire de ses Voyages, écrit en langue anglaise, a été publié dans la série des documents historiques de la Prince Societ 3 .

7. Cette Histoire du Canada, restée en manuscrit à la Bibliothèque Nationale de Paris, fut publiée à Québec par les soins de la Société Littéraire et Historique de cette Ville en 1840. 
archives publiques et privées. Il en a fait la publication dans son Histoire de la Colonie Française en Canada dont il a commencé la publication en 1865, mais qu'il a malheureusement discontinuée après le troisième volume. Son récit de l'exploit de Dollard et de ses compagnons est en même temps le plus complet et le plus attachant que nous connaissions; l'historien Parkman s'en est largement inspiré dans son Old Regime in Canada qui fait partie de sa série d'ouvrages historiques.

Comme il n'entre pas dans le cadre de cet article de faire un nouveau récit de l'exploit du Long-Sault, vu que les documents ci-dessus indiqués y pourvoient amplement, il ne s'agit ici que de situer indubitablement l'endroit où il s'est accompli afin qu'on puisse vénérer, sans être assailli de doutes, le sol qui a bu le sang de ces sauveurs de la patrie.

Etablissons tout d'abord la genèse du projet et la route suivie par ces braves pour le réaliser:

"Sur la fin d'avril, écrit Dollier de Casson, M. Daulac, garçon de cœur et de famille, lequel avait eu quelque commandement dans les armées de France, voulant faire ici quelque coup de sa main et digne de son courage, tâcha de débaucher quinze ou seize Français, pour les mener en parti au dessus de cette ile, ce qu'on n'avait point encore osé tenter. Il trouva de braves garçons qui lui promirent de le suivre si M. de Maisonneuve le trouvait bon. Daulac proposa la chose et il eut son agrément, ensuite chacun se disposa à partir, mais auparavant ils firent un pacte de ne pas demander quartier et se jurèrent fidélité sur ce point."

Ainsi, Dollard n'est pas un aventurier ni un écervelé. C'est "un garçon de cœur et de famille lequel avait eu quelque commandement dans les armées de France" et nos historiens ajoutent même qu'il commandait la garnison de Ville-Marie. Son dessein n'était pas d'aller attaquer les Iroquois au retour de leurs chasses d'hiver pour s'emparer de leurs fourrures ainsi que certains détracteurs ont osé l'insinuer; il veut mener ses compagnons en parti de guerre contre l'ennemi irréductible "ce qu'on n'avait point encore osé tenter". Ce dessein reçoit une confirmation expresse dans le testament de Jean Valets, l'un des compagnons, passé devant Me Basset, notaire, le 18 avril 1660, dans lequel il déclare quel est le but de cette expédition 
en disant: "Lequel désirant aller en party avec le Sr. Dollard, cour(ir) sur les petittes bandes hiroquoises et nos ennemis". De son côté la Mère de l'Incarnation écrit à son fils: "Dix-sept braves Français, volontaires de Montréal, prirent le dessein de se hasarder pour aller faire quelque embuscade aux Iroquois". Mais à cause des règlements sévères établis depuis 1658 par M. de Maisonneuve afin de protéger ses administrés, il fallait avant tout obtenir son assentiment avant d'en tenter l'aventure et le projet parut tellement désintéressé et sublime au fondateur de Ville-Marie, qu'il y acquiesça. Croit-on qu'il l'eût approuvé s'il eût soupçonné qu'il s'agissait d'un complot de bandits?

Établissons maintenant la route que nos conjurés suivent pour atteindre leur destination; c'est la Relation des Jésuites pour l'année 1660 qui va nous l'indiquer. "Ayant gagné le Lac Saint Louis, qui est au dessus de l'île de Montréal, ils détournent à droite, entrent dans la Rivière qui mène aux Hurons ${ }^{8}$ et vont se poster au dessous du Sault de la Chaudière pour y attendre les chasseurs iroquois qui selon leur coutume le doivent passer file à file en retournant de leur chasse d'hiver". Il y a lieu de faire ici une mise au point: les Hurons qui ont raconté l'aventure du Long-Sault au Père Lallemant, rédacteur présumé de cette Relation à Québec, n'ont sans doute pas précisé de quel "sault" il s'agissait et le bon missionnaire, qui connaissait le "sault" de la Chaudière pour y avoir souvent passé, en a inscrit le nom sans s'arrêter à songer qu'il eût été humainement impossible à Dollard et ses compagnons d'atteindre cet endroit en deux jours de montée du courant avec la rencontre des glaces et les nombreux "portages" à effectuer. Notons en outre que, dans ce récit, il est dit qu'il faut descendre ces rapides "file à file", c'est à dire à la file indienne, afin de ne pas chavirer, preuve évidente qu'il ne peut s'agir du "sault" de la Chaudière qui est trop impétueux, mais bien du "passage infaillible" du Long-Sault à Carillon.

Le récit de Dollier nous donne plus de précision sous ce rapport en disant: "Ils arrivèrent au pied du Long-Sault où, trouvant un petit fort sauvage, nullement flanqué, entouré de méchants pieux qui ne valaient rien, commandé par un coteau voisin, ils se mirent dedans

8. C'est la Rivière Ottawa que certains auteurs anciens nomment également Grande Rivière. 
n'ayant pas mieux" et, de son côté, Vachon de Belmont nous dit: "A u pied du Long Sault, il trouva un méchant fort de pieux commandé d'un coteau et se jette dedans".

D'autre part, la Relation de 1660 ajoute: "comme l'ardeur du combat était grande et les attaques presque continuelles, la soif pressait plus nos gens que l'Iroquois. Il fallait essuyer une grêle de plomb et aller à la pointe de l'épée puiser de l'eau à la Rivière qui était à deux cents pas du fort, dans lequel on trouva enfin, à force de fouir, un petit filet d'eau bourbeuse, mais si peu que le sang découlait des veines des morts et des blessés bien plus abondamment que l'eau de cette source de boue."

Nous voici donc quelque peu renseignés sur l'endroit où la vaillante phalange de Ville-Marie va rencontrer les guerriers Iroquois qui descendent la Rivière Ottawa pour opérer leur fonction avec ceux qui sont venus camper dans les îles du lac Saint.Pierre par la voie du Richelieu. C'est au pied du rapide, à une douzaine de milles de l'île de Montréal et le "méchant fort" qu'ils y trouvent pour se retrancher avait été construit par des Algonquins l'année précédente, près d'un coteau voisin et à deux cents pas (soit environ cinq cents pieds) de la rivière. L'endroit est bien choisi comme lieu d'embuscade, car les canotiers qui montent le courant doivent mettre pied à terre en ce lieu pour y faire un "portage" afin de contourner le rapide, alors que ceux qui le descendent sont tenus de naviguer "file à file" en longeant le rivage par le "passage infaillible" afin de ne pas chavirer dans les eaux du sault, les voyageurs qui "montent" et ceux qui "descendent" la rivière ayant pour objectif la petite baie qui tourne en remous au pied du rapide.

On nous dira peut-être: "Mais sur quoi vous appuyez-vous pour dire que la route suivie par Dollard et ses compagnons était celle de la rive nord de l'Ottawa alors que la navigation sur l'eau calme et le portage au long des rapides pouvaient se faire également par la rive sud où l'Équipe géologique a trouvé les traces du fort qui ré. pondrait beaucoup mieux à l'idée qu'on peut se faire d'un siège de vingt six jours?"

La réponse est bien simple.

Si les Relations des Jésuites, les historiens, les missionnaires, les coureurs de bois, les voyageurs, les chefs d'expédition et la plupart de ceux qui ont laissé des Mémoires n'ont pas cru nécessaire de 
donner des précisions sur la route qu'ils suivaient en se rendant vers les territoires du nord et de l'ouest, c'est qu'il n'y en avait qu'une qui était généralement suivie, depuis Champlain qui y avait perdu son astrolabe en 1613, jusqu'aux Bourgeois de la Compagnie du NordOuest en $1825^{\circ}$. On remontait la rivière Ottawa en faisant portage sur sa rive nord en face des rapides et on la descendait par le "passage infaillible" le long de cette même rive. C'est également cette route que suivaient les Iroquois dans leurs chasses d'hiver sur les rivières du Lièvre et de la Petite Nation; on se rendait par la rivière Ottawa jusqu'à la Mattawa où l'on bifurquait vers le nord pour aller à la baie d'Hudson ou vers l'ouest pour les Grands Lacs. C'est également elle que les autorités militaires et civiles ont officiellement reconnue plus tard en y creusant le canal de Carillon à Grenville, lorsque la nécessité s'en est fait sentir, ainsi que la Compagnie de Chemin de fer Montreal and Bytown pour y faciliter le transport des marchandises.

Le journal très minutieux et circonstancié de l'expédition du chevalier de Troyes à la baie d'Hudson, accompli par voie de terre en 1686, nous en fournit la preuve précise en même temps qu'il situe exactement le théâtre du combat du Long Sault ${ }^{10}$. Il conduisait un parti de trente soldats des troupes régulières et soixante-dix hommes entraînés à ces voyages difficiles, assisté de Jacques Lemoyne de Sainte-Hélène, Pierre Lemoyne d'Iberville, Paul Lemoyne de Maricourt, Zacharie Robutel de la Noue, beau-frère des trois précédents, et de Pierre Allemand, pilote renommé et commissaire des vivres, avec le Rév. Père Silvy en qualité d'aumônier. Les extraits suivants du journal de cette expédition, commencée à Montréal le 30 mars 1686, nous conduisent jusqu'à Mattawan qui fut atteint le 12 mai suivant; nous en respectons le style et l'orthographe.

9. Dans un article publié au mois de janvier 1951 du Canadien Geographical Journal, George R. Rumney l'indique clairement et l'accompagne d'illustrations comme étant la route suivie, par la rivière Ottawa et le Lac Nipissing, pour se rendre en canot vers les territoires de l'Ouest.

10. Le manuscrit original de ce précieux document fait partie de la collection Clairambault. Il est catalogué sous le No $1026 \mathrm{a}$ la Bibliothèque Nationale de France a Paris et il a été publié pour la première fois par les soins de l'abbé Ivanhoë Caron à Beauceville en 1918 . 
"Le lendemain, dernier jour du mois, plusieurs de nos gens enfoncèrent dans la glace qui se trouva si mole que cela obligea le Sr. de St. Germain à renvoyer ses bœufs qui enfoncèrent de nouveau à vingt pas du camp, avec leurs traines et furent sauvez par le secours que l'on leur donna... Je campé dans un anse où je trouvé le sieur de la Forest, capitaine dans les troupes que commande Monsieur de la Salle, qui allait aux Illinois avec un équipage de trois canots, tenant notre route jusques au Mattaouan.

Le premier avril, je séjourné, tout mon équipage n'ayant pu suivre le jour précédent, me contentant de faire prendre le devant à ceux qui se trouvèrent en état de continuer la marche, qu'un dégel rendit extrêmement pénible, estant obliger dans le chemin de traisner leur bagage, marchand dans la neige fondue jusques aux jambe. Ils furent camper dans l'isle de Carillor ${ }^{11}$ où un gros arbre poury tomba sur un de leurs canots et l'écrasa de sa chutte, ce qui m'obligea, sur l'avis que j'en eu, de commander du monde pour aller quérir un autre au bout de l'isle.

Le deuxième avril, je me rendis avec le reste de mon détachement à l'isle de Carillon accompagné du R.P. Silvie, qui me joignit ce jour la... Le troisième jour d'avril, je séjourné à cause de la pluie mais aiant gelé toute la nuit, je fus camper le $4 \grave{e}$, au pied du Long Sault. Je marchais avec une épée amenchée dont je sóndais la glace fine qui s'estait faite la nuit précédente... faisant fond sur la grosse glace que je m'imaginais trouver dessous, mais l'usage fréquent que je fis de mon épée me fist connaítre mon erreur et le danger où j'estais qui m'obligea de quitter le canal ${ }^{12}$ où je marchais comme par le plus court, et de continuer la route le long de terre. Le R.P. Silvie me suivait pas à pas, et courant les mêmes risques. Nous vîmes au bout du Long-Sault, les vestiges où dix sept français soutinrent, pendant les antiennes guerres des Iroquois, l'effort de sept cents de ces barbares dont ils tuèrent et blessèrent un nombre très considérable...

Le cinquième jour d'avril, ceux qui estaient restez derrière arrivèrent au camp, où nous séjournames pour raccommoder nos canots,

11. Ce nom lui vient de la Seigneurie concédée à Philippe Carrion du Fresnay en 1670 et du privilège de traite de pelleterie qui lui fut accordé sur la rivière Ottawa en même temps. Il s'est par la suite étendu à la paroisse et au village du même nom.

12. C'est-à-dire la baie dormante aboutissant au "passage infaillible" où l'eau était plus calme. 
les régommer et faire perches et avirons, estant obligés à toutes ces précautions d'autant que nous estions au pied d'un rapide qui ne gèle jamais et où il fallait traisner et percher. Ainsi le Sr. de St. Germain que j'avais envoié reconnaistre le bordage par dedans le bois, m'aiant assuré que le portage estait impossible, cela me fist resoudre de monter le rapide comme en plain esté, c'est à dire dans l'eau jusqu'à la ceinture.

Le neufvième avril, messieurs de Ste hélène et d'hyberville, accompagnez d'habiles canotteurs, commencèrent à monter le Long Sault ${ }^{13}$. Ils portèrent leurs bagages au-dessus du premier rapide qui est à une lieue de la pointe du lac du nom des Deux Montagnes ${ }^{14}$ qui commence au bout de l'isle de Montréal et finit au pied du Long Sault...

Le dixième avril, je fis partir les canots qui estaient restez au camp, et m'acheminé dans le bois le long de l'eau avec le P. Silvie et ceux qui n'estaient pas nécessaires pour aider à monter les canots dont plusieurs furent endómmagés... L'unzième avril je fus dans mon canot alège aiant avec moi messieurs d'hyberville et de St. Cermain pour visiter le chemin suivy de trois canots et du monde nécessaire pour en faire un (chemin) dans un portage de la longueur d'une portée de fusil ${ }^{15}$.

Le dix neufviesme, nous décampâmes de fort bonne heure pour aller à un lieu nommé la chaudière à environ neuf lieues de la $\grave{a}^{16}$, ce que nous ne pûmes faire à cause qu'il fallut s'arrester pour raccommoder nos canots. Nous passâmes la rivière du lièvre ${ }^{17}$ pour $y$ prendre un (canot), qui, y estait, qu'il fallut regommer tout à neuf aiant passé là l'hyver. Nous fumes camper à deux lieues plus haut où tous les canots à cinq ou six près nous vinrent joindre le lendemain. Pendant toute cette route nous avions fait plusieurs fois rencontre de troupes d'Iroquois

13. C'est-à-dire les trois rapides qui se trouvent dans l'espace aujourd'hui compris entre Carillon et Grenville.

14. C'est l'endroit que le chevalier de Troves a précédemment indiqué comme étant celui du fort ou dix sept Français ont subi l'assaut de sept cents Iroquois.

15. C'est le deuxième portage en montant la rivière, c'est-à-dire celui qui était nécessaire pour éviter la Chute à Blondeau. Cette chute a été presque totalement "noyée" en 1830 par la construction de la digue de Carillon. Ottawa.

16. C'est la chute de la Chaudière située entre les villes actuelles de Hull et

17. Remarquons ici que la rivière du Lièvre coule sur la rive nord de l'Outaouais. de sorte que la route suivie était bien située sur la rive nord. 
Chrestiens qui nous pressoient charitablement de séjourner dans des endroits où ils offraient de nous régaler, ce que ne pouvant leur accorder pour faire plus de diligence...

Le douzième may, nous allâmes à Mataouan qui signifie en langue sauvage "fourche de rivière", $y$ en ayant effectivement une en cet endroit dont la gauche, qui est au sud, est le chemin des Outaouais et la droitte, qui est au nord, par conséquent mon chemin, conduit aux Themiskamingue... Je disné avec le Sr. Juchereau qui venait de Michilmakina, et s'en allait à Québeck en grande diligence, porter des nouvelles à Mr. le marquis de Denonville."

Cette citation que nous avons copieusement reproduite à dessein suffira-t-elle à convaincre les plus incrédules que la route suivie par les Iroquois pour monter à leurs terrains de chasse des rivières de la Petite Nation, du Lièvre et de la Gatineau, de même que par les découvreurs, missionnaires, coureurs de bois et militaires pour se rendre à Michillimakinac et dans la région des Grands Lacs, ainsi que le "passage infaillible" pour en descendre en lisière des rapides $\mathrm{du}$ Long-Sault, longeaient la rive nord de la rivière des Outaouais, et que pour y rencontrer infailliblement l'ennemi, c'est là que Dollard et ses compagnons devaient aller se poster?

Suffira-t-elle à leur prouver que "les vestiges où dix-sept Français soutinrent... l'effort de sept cents de ces barbares" se trouvaient au pied du dernier des trois rapides du Long Sault où le chevalier de Troyes s'en fut camper le 4 avril 1686, jour où il visita, en compagnie du Rév. Père Silvy, le lieu de ce combat et où il séjourna jusqu'au 10 avril pour regommer ses canots et tailler des perches et avirons afin de remonter les rapides?

Si ce témoignage, qui est d'ordre historique et traditionnel, ne leur semble pas assez concluant, nous leur en fournirons deux autres, d'ordre scientifique ceux-là, afin de vaincre leur entêtement: le premier basé sur l'hydrographie et le second sur la topographie des lieux.

L'abbé Arthur Guindon, prêtre de Saint-Sulpice, a fait une étude spéciale de l'hydrographie des deux rives du Long-Sault, tant sur place qu'au moyen des cartes dressées avant et après la construction des canaux de Carillon à Grenville et du barrage qui a pratiquement fait disparaître la Chute à Blondeau. Or il a publié, 
dans le journal Le Devoir en date du 6 août 1910, un article très concluant, accompagné d'un graphique explicite où il démontre à i'évidence que, surtout avant la construction de la digue de Carillon, les eaux du rapide se précipitaient impétueusement de la rive sud vers la rive nord et que les "draveurs" des "cages de bois" avaient toutes les difficultés possibles à empêcher leurs radeaux d'aller se briser sur les rocs de la rive sud à cet endroit.

A l'aide des documents cités plus haut (sauf la relation du chevalier de Troyes qui n'avait pas encore été publiée à cette époque) le savant abbé localisait en outre l'endroit qu'occupait le fort de Dollard des Ormeaux au haut d'une petite rue transversale à la route qui longe la rive nord de la rivière à Carillon et $\mathrm{y}$ faisait ériger une croix sur la propriété Séguin, à l'intersection de l'ancien chemin de fer qui conduisait du quai de Carillon à Grenville à cet endroit ${ }^{18}$. Que le service Géologique du Canada y vienne faire des fouilles; il ne manquera pas d'y trouver les traces des palissades du fort des Algonquins qui n'ont pas été complètement bouleversées par la construction de cette voie ferrée.

Examinons maintenant la topographie des lieux et voyons si les relevés de palissades faits sur la rive sud de la rivière Ottawa peuvent s'apparenter au fort de Dollard des Ormeaux:

10. La rive sud de la rivière est abrupte et pierreuse à l'endroit où s'élevait le fort de pieux dont on a relevé les traces; les rapides de la Chute à Blondeau se jetaient sur la côte avec une telle impétuosité, avant la construction de la digue de Carillon, que les canotiers ne pouvaient songer à s'y engager au risque de se perdre corps et biens.

Mais le "passage infaillible" où les canotiers pouvaient descendre la rivière en sécurité, entre les aspérités des rapides et la côte, ne se trouve qu'au long de la rive nord et se termine à Carillon; c'est là que devait "infailliblement" passer la troupe iroquoise que Dollard avait décidé d'affronter.

2o. L'enceinte palissadée dont l'équipe géologique a relevé les vestiges sur la ferme Ross représente une ellipse assez régulière

18. M. l'abbé Guindon a continué ses études sur cette question par la suite et les a résumées dans une brochure intitulée Les Trois Combats du Long Sault publiée en 1923. 
de soixante pieds sur soixante-dix; son emplacement a fait l'objet d'un choix stratégique et sa construction, surtout du côté de la rivière, a été faite avec un soin méticuleux. On y reconnaît l'art consommé des Iroquois.

Mais le "méchant fort construit par les Algonquins l'année précédente" était plus ou moins un abri de fortune; les palissades en étaient plutôt branlantes; il a fallu les assujétir par le haut avec les arbres voisins et en remplir les interstices avec des pierres et de la terre.

3o. Ce "méchant fort des Algonquins" était "domine" par une colline avoisinante, bien qu'il n'en fût pas assez rapproché pour être exposé au feu plongeant des mousquets à courte portée de cette époque.

Mais le fort de la ferme Ross s'élevait au contraire sur un terrain d'un niveau à peu près uniforme; de fait, c'est plutôt son emplacement qui domine le terrain avoisinant.

4o. Le fort de Dollard des Ormeaux était situé à "deux cents pas" soit environ cinq cents pieds de la rivière où il fallait aller puiser de l'eau sous le feu de l'ennemi.

Mais celui de la ferme Ross n'était qu'à environ cent quatre vingt-dix pieds de la rive sud de cette même rivière.

5o. La Relation des Jésuites nous dit "qu'à force de fouir" à l'intérieur du fort on finit par y trouver "un petit filet d'eau bourbeuse".

Mais le sol de la rive sud étant de tuf, ne permettait même pas d'y "fouir" avec quelque succès.

6o. De plus, un petit ruisseau coule en arrière de l'emplacement du fort de la ferme Ross, à trente pieds seulement de distance de la palissade sud, chose qui aurait apporté une solution facile au problème d'approvisionnement d'eau de Dollard et de ses compagnons si leur fort avait été situé de ce côté de la rivière.

Il nous faut donc en venir forcément à conclure que les vestiges de palissades découverts sur la rive sud de la rivière Ottawa ne peuvent s'apparenter en aucune manière avec le fort de Dollard des Ormeaux. 
Mais alors ?... Que serait donc cette enceinte fortifiée dont le Service Géologique semble avoir indiscutablement établi la découverte sur la ferme Ross à cinq milles en aval de Hawkesbury? M.T.E. Lee qui a rédigé le rapport de cette exploration est un géologue averti et la réputation du Dr Marius Barbeau dont il a sollicité la collaboration est solidement établie comme anthropologue de grand mérite. Nous avons soigneusement étudié ce rapport, ainsi que les cartes préparées pour sa compréhension, et nous sommes d'avis que ces savants ont fait une découverte intéressante à cet endroit mais... qu'ils se sont trop hâtés de l'identifier avec l'exploit de Dollard et de ses compagnons en se basant sur une vague tradition locale ${ }^{19}$ à savoir "qu'une grande bataille y avait eu lieu entre les Français et les Indiens", sans tenir suffisamment compte des données précises de I'histoire à ce sujet.

Après étude des textes historiques et de la topographie des lieux, nous en sommes venus à risquer la conclusion que le fort dont $M$. Lee a si minutieusement relevé les vestiges à un tiers de mille plus bas que le ruisseau du Petit Rideau sur la rive sud de la rivière Ottawa, aurait plutôt été construit à cet endroit par le redouté chef onnontague "Chaudière Noire" avec toute la science stratégique des guerriers iroquois, pour en faire le refuge de sa guerre d'embuscade sur cette artère de communication avec les "pays d'en haut". Ce n'est qu'une théorie si l'on veut, mais elle peut s'établir encore plus logiquement que l'hypothèse de la ferme Ross sur les faits suivants qui sont relatés dans les récits historiques de cette époque:

La Chaudière Noire et sa bande guerroyaient sur les deux rives de l'Ottawa et jusqu'au fleuve Saint-Laurent à l'entrée du lac Ontario. S'ils étaient trop vivement pressés sur la rive nord de l'Ottawa, où ils opéraient plus volontiers vu que c'était l'artère de communication avec les "pays d'en haut", ils traversaient la rivière afin de s'enfermer dans cette forteresse dont les plus solides retranchements se dressaient vers la rive, car l'attaque était plus logiquement à craindre de ce côté.

Nous lisons en effet dans Bacqueville de la Potherie ${ }^{20}$ et dans

19. Ils disent la tenir des pêcheurs de l'endroit.

20. Histoire de l'Amerique Septentrionale (4 vol., Paris, 1723), III: 159 a 161. 
Charlevoix ${ }^{21}$ qu'après avoir exercé ses déprédations et ses meurtres à la Pointe-aux-Trembles et à La Chesnaye au mois de juillet 1692 , la troupe de la Chaudière Noire se retira vers les rapides du Long Sault et que M. de Callières, alors gouverneur de Montréal, les y fit poursuivre par le chevalier de Vaudreuil assisté de Du Plessis Faber et Villedonné à la tête de quatre cents hommes, dont une centaine de sauvages de la Montagne et du Sault Saint-Louis. Afin d'envelopper l'ennemi, M. de Vaudreuil laissa sa flotille de canots en garde de cent hommes sur la rivière à une certaine distance avant d'arriver au Long-Sault et prit le chemin des bois avec le gros de son armée en faisant converger son aile gauche vers la rivière. Sa tactique lui réussit pleinement car en attaquant le fort des Iroquois dans sa partie la plus vulnérable, il les accula tellement à la rivière que ceux-ci n'eurent pas d'autre ressource que de se jeter à la nage ou si précipitamment dans les canots que la plupart furent tués ou noyés ${ }^{22}$. L'habile Chaudière Noire fut au nombre de ceux qui réussirent à s'échapper à la nage; mais s'il était brave, habile et vigoureux, il ignorait les principes de la chevalerie française, car il abandonnait Madame Chaudière Noire avec huit autres femmes, cinq hommes et cinq enfants aux mains des vainqueurs qui délivraient, de leur côté, neuf prisonniers français et trois enfants sauvages dont il s'était emparé à la Chesnaye quelques jours auparavant ${ }^{23}$.

Telle est, à notre avis, l'histoire du "grand combat entre les Français et les Indiens" qui s'est livré sur la rive sud de la rivière Ottawa, à l'endroit découvert et décrit par M. Lee, mais qu'il a trop hâtivement tenté d'identifier avec l'immortel exploit accompli par Dollard des Ormeaux et ses valeureux compagnons à Carillon.

Alors... pourquoi s'évertuer, après trois siècles de distance, à

21. Histoire et Description Générale de la Nouvelle-France (4 vol., Paris, 1744), II: 114 à 116.

22. Arthur Guindon, p.s.s. Les Trois Combats du Long Sault, (Montréal, 1923).

23. Dans un article publié par la Revue Nationale de la Société Saint-Jean-Baptiste, Gérard Malchelosse a raconté les actes de piraterie de la Chaudière Noire sur l'Ottawa. C'est un jeune guerrier Algonquin de dix-huit ans, du nom de Ki8et qui y mit fin à l'été de 1697 en tuant le redoutable chef dans une embuscade à la baie de Quinté et en scalpant sa chevelure, suprême avanie qui l'humiliait au point de dire: "Moi qui ai fait trembler la terre, je péris de la main d' un enfanl". (Cf. Les Ursulines des Trois-Rivières, depuis leur établissement jusqu'à nos jours, (4 vol., Trois-Rivières, 1888-1911), 1: 21. 
toujours mettre en doute les témoignages d'historiens de mérite et prétendre que leurs constatations ne valent rien tandis que nous seuls, grâce aux lumières des temps modernes, sommes gardiens de la Vérité?

Songeons-nous, en prenant cette attitude, que nos descendants viendront peut être déclarer à leur tour, avec preuves en mains, que nous n'étions que des buses et, peut-être même, que les faits les plus héroïques de notre histoire ne sont guère que des racontars pour les petits enfants? C'est un peu ce qu'on a parfois tenté de faire au détriment de Dollard et de ses compagnons.

Ayons donc du respect pour les documents authentiques et les traditions ancestrales bien établies. Nous y trouverons un réconfort que des hypothèses plus ou moins justifiables ne réussiront jamais à remplacer.

Victor MORIN, de l'Institut d'histoire de l'Amérique française 